\title{
AUTOMAÇÃO RESIDENCIAL COM UM SISTEMA CONTEXTUAL PARA CONTROLE DE EQUIPAMENTOS DOMÉSTICOS (SCED)
}

\section{HOME AUTOMATION WITH A CONTEXTUAL SYSTEM FOR HOUSEHOLD EQUIPMENT CONTROL (SCED)}

\author{
Fabio Fonseca Barbosa Gomes¹, Igor González Pimenta², Leandro Fernandes de Oliveira ${ }^{3}$
}

\begin{abstract}
RESUMO
Muitos dispositivos eletrônicos em uso, atualmente, trazem dificuldades para os usuários. Os controles remotos tradicionais possuem uma série de problemas como o diferente conjunto de botões, falta de bateria ou pilha além da grande quantidade de controles necessários para operar diversos dispositivos diferentes. Quanto mais pessoas tiverem Smartphones, a possibilidade de usá-los como controles remotos universais para facilitar o uso de dispositivos eletrônicos domésticos se torna mais evidente. Para isso é necessário o desenvolvimento de um aplicativo que precisa ser sensível ao contexto, porque ele se adaptará a condições variáveis, como proximidade do dispositivo, conjunto de dispositivos e status dos dispositivos. Este artigo relata a implementação e testes funcionais e de usabilidade do SCED, um aplicativo que visa controlar um conjunto de dispositivos eletrônicos em casa. Foi construído com base em resultados de pesquisas anteriores sobre a integração de dispositivos eletrônicos usando componentes baratos como Raspberry Pi e microcontrolador Arduino. Para uma validação preliminar foram realizados alguns testes com voluntários e estão presentes os resultados de usabilidade.
\end{abstract}

PALAVRAS-CHAVE: Automação Residencial. Controle Universal. Contexto.

\begin{abstract}
So many electronics devices in use at homes nowadays bring a sort of difficulties to users. A lot of remote controls, different set of buttons, battery outage and lost remote controls make their use sometimes problematic. As more people have Smartphones, use them as universal remote controls could make the use of domestic electronics devices easier. Such application needs to be context-aware, because it will adapt to variable conditions like device proximity, set of devices, and devices status. This paper reports implementation and functional and usability tests of the SCED, an application that aims to control a set of electronic devices at home. It was built based on former research results about integrating electronic devices using cheap components like Raspberry Pi and Arduino microcontroller. For a preliminary validation were conducted some tests with volunteers and are presents usability results.
\end{abstract}

KEYWORDS: Residential Automation. Universal Control. Context.

\footnotetext{
${ }^{1}$ Graduado em Sistemas de Informação pelo Centro Universitário Estácio da Bahia, Mestre em Sistemas e Computação pela Universidade Salvador. Docente do Centro Universitário UNIRB, Centro Universitário Dom Pedro II e Fundação Visconde de Cairu.

${ }^{2}$ Graduado em Sistema de Informação pela Faculdade Ruy Barbosa, mestre em Sistemas e Computação pela Universidade Salvador. Docente di - SENAI - Departamento Regional da Bahia, analista de sistemas - ICATU BAHIA, professor da Faculdade Regional da Bahia - UNIRB e professor mestre da Faculdade Maurício de Nassau - Salvador.

${ }^{3}$ Graduado em Sistemas de Informação e pós-graduado em Engenharia de Software pela Universidade Salvador - UNIFACS.

Professor titular do SENAI.
} 


\section{INTRODUÇÃO}

As inovações relacionadas aos recursos tecnológicos, miniaturização dos componentes eletrônicos, aprimoramento das redes sem fio e crescente oferta de serviços Internet geram novas oportunidades e desafios para o desenvolvimento de aplicativos para a automação residencial (BÖHMER et al., 2013). Isto ocorre na mesma velocidade em que o hardware evolui na área de sensores e atuadores, aumentando o número de usuários com dispositivos móveis. Além disso, cada vez mais os smartphones e tablets ganham poderosos recursos computacionais e passam a dispor de um maior número de aplicativos (VIEIRA et al., 2011).

Um dos campos que pode se beneficiar desta evolução na tecnológica é a domótica ou automação residencial. Neste tipo de ambiente existem apenas dois tipos de dispositivos elétricos: aqueles que são ligados através dos botões e outros que funcionam a través de controles remoto. Ainda assim, apesar do uso de controles remotos ser melhor que o uso de botões analógicos, esta tecnologia não possui um padrão definido entre os fabricantes e muitos aparelhos possuem controle remoto único, obrigando as pessoas a terem vários controles remotos em suas casas (SU; CHIANG, 2014).

Uma forma de solucionar os problemas relatados converter o smartphone em um controle remoto universal, através da utilização de uma aplicação para dispositivos móveis (App). Este App proverá uma tela humano-computador padronizada para todos os equipamentos, assim como estenderá o recurso de controle remoto aos equipamentos acionados por interruptores e botões tradicionais. A aplicação deverá ter uma semântica, para reduzir a necessidade de configuração explícita e formação dos acionamentos. Além da App, necessitará de um módulo de gerenciamento que faça a efetiva interação com os equipamentos.

Para construir esta solução foi implementada uma aplicação sensível ao contextual denominada SCED (Sistema Contextual para Controle de Equipamentos Domésticos), integrado à plataforma desenvolvida por (PIMENTA et al., 2015). A plataforma foi expandida com o acréscimo de mais uma forma de interação. A validação da solução foi realizada através de uma simulação de uso. A simulação desenvolvida neste trabalho teve como cenário a residência de uma pessoa que segue uma rotina diária, com pequenas variações em determinados horários. Esta simulação foi capaz de englobar todas as ações do sistema podendo realizar sua validação.

Como contribuições deste trabalho, podem ser destacadas: (i) a inclusão de mais uma forma de interação que prevê a integração de diferentes aparelhos eletrônicos em um ambiente residencial dentro da plataforma criada por (PIMENTA et al., 2015), independente do seu modelo e/ou marca; (ii) o desenvolvimento de uma aplicação que acessa uma ontologia com a finalidade de ser um controle remoto universal, que se adapte de forma fácil e dinâmica às necessidades de interação das pessoas e do fabricante dentro do ambiente; e (iii) a construção de uma tela que se molda automaticamente em função dos dispositivos eletrônicos existentes no cenário de interação.

O restante deste artigo está dividido nas seguintes em seções: características funcionais dos controles remotos; principais aplicativos de controle remoto disponíveis no mercado; a apresentação do aplicativo que valida o modelo proposto; avaliação da solução; a discussão dos resultados encontrados após a realização da simulação; a análise de alguns trabalhos correlatos; e as conclusões e trabalhos futuros.

\section{CONTROLES REMOTOS UNIVERSAIS}

Equipamentos eletrônicos apresentam diferentes codificações, por isso os controles remotos universais são dispositivos que precisam ter o conhecimento de diversas formas de como utilizá-los. Nos dias atuais, existem diversos meios de transmissão, como radiofrequência ou infravermelho, entretanto, os mais modernos usam tecnologias como Zigbee, Bluetooth ou Wi-Fi.

Os controles remotos podem ser divididos em duas categorias: (i) os multimarcas, que já vêm uma configuração prévia para a utilização de determinados apareIhos de uma série de fabricantes como, por exemplo, os televisores e home theaters da Samsung, LG e Philips; e (ii) os denominados programáveis, que podem se comunicar com qualquer aparelho de acordo com a programação realizada pelo usuário (DESAl et al., 2002).

A maior dificuldade dos controles remotos universais se consolidarem como uma alternativa amplamente utilizada, apesar de estarem no mercado há bastante tempo, é que os aparelhos eletrônicos são comprados com os seus controles próprios. Além disso, muitas mar- 
cas encapsulam os seus códigos de acesso, impedindo a utilização dos controles remotos universais (DVORAK et al., 2019).

Fazendo uma análise em ambiente residenciais, percebe-se que ainda existe uma grande quantidade de controles remotos, sendo que cada um é usado para um aparelho diferente. Esta situação causa problema para indivíduos usam muitos dispositivos eletrônicos, principalmente no momento de localizar o mesmo para ser usado. Como consequência, a sua utilização e eficiência são drasticamente reduzidas (DVORAK et al., 2019).

Outras dificuldades analisadas são: o número excessivo de teclas; a falta de padronização; as limitações para a personalização ao usuário; e a dificuldade para realizar configurações. Em função das dificuldades citadas, surgiu a necessidade de utilizar uma tecnologia que unifique os diferentes controles remotos de maneira contextual e que esteja disponível grande parte do tempo para os usuários. Desta maneira, a utilização do smartphone como controle remoto se tornou uma tendência, já que estes aparelhos geralmente estão presentes no cotidiano de qualquer pessoa (SATRIA, 2015).

\section{TRABALHOS CORRELATOS}

A literatura apresenta aplicações com funcionalidades similares a plataforma proposta neste trabalho. Desta maneira, foram encontradas algumas propostas para a criação de plataformas de automação residencial, tais como: Piyare (2013), Dvorak et al. (2019), Zhong et al. (2011), Vikram (2017).

O trabalho de Zhong et al. (2011) utiliza um aplicativo para smartphone chamado HouseGenie que controla outros dispositivos a distância. A comunicação entre eles é realizada utilizando uma plataforma dividida em quatro camadas: (i) camada de tela, onde funciona o controle universal; (ii) camada de serviços, que disponibiliza um middleware para o controle e tratamento de aspectos contextuais; (iii) camada de dispositivos, definidas pelos dispositivos eletrônicos do ambiente; e (iv) camada adaptativa, na qual estão as tecnologias de rede utilizadas pelos dispositivos eletrônicos.

O trabalho de Piyare (2013) apresenta uma plataforma chamada de Ubiquitous Home Control and Monitoring System using Android que possibilita aos usuários a comunicação com os dispositivos de uma smart home, através de um aplicativo móvel dentro ou fora da resi- dência, com o uso da Internet. Para o funcionamento desse aplicativo, foi definida uma plataforma dividida em três camadas, cada uma com funcionalidades especificas, tais como: (i) a Remote Enviroment, responsável pelos programas instalados no dispositivo móvel; (ii) a camada Home Gateway, que realiza a intermediação da troca de mensagens entre o aplicativo e os dispositivos eletrônicos através de um atuador; e (iii) a camada de Home Enviroment, que é responsável por executar as ações junto aos dispositivos eletrônicos.

Dvorak et al. (2019) propuseram uma solução utilizando uma placa PCB, juntamente com um aplicativo que, combinados, formariam uma plataforma que funciona como um controle remoto universal, priorizando dispositivos que não possuem acesso a controle remoto. $O$ objetivo era que esses aparelhos tivessem a possibilidade de ser controlados remotamente.

Vikram et al. (2017) propuseram uma plataforma residencial que tem a capacidade de medição de temperatura, umidade, gás, além do controle dos dispositivos móveis do ambiente através do uso de um controlador central e ele pode controlar os sensores no ambiente via smartphone. A tela não pode ser moldada, porém apresenta todas as opções necessárias para o usuário controlar a residência.

De acordo com o levantamento realizado, foi possível observar que existem propostas nas quais os dispositivos móveis se conectam diretamente com os atuadores, Satria et al. (2015), Roy e Roy (2014), enquanto outras utilizam um middleware para tratar a interoperabilidade entre as diferentes tecnologias Dvorak et al. (2019), Zhong et al. (2011) e Vikram et al. (2017).

A Tabela 1 apresenta um resumo comparativo entre as propostas de controles remotos universais encontrados na literatura que utilizam smartphones como forma de interação.

Pode-se perceber que o SCED possui duas vantagens em relação aos outros aplicativos: (i) detecta automaticamente os dispositivos eletrônicos e (ii) disponibiliza telas personalizadas de acordo com o dispositivo eletrônico escolhido pelo usuário. 
Tabela 1. Comparação do SCED com outras propostas encontradas na literatura

Table 1. Comparison of SCED with other proposals found in the literature

\begin{tabular}{|c|c|c|c|c|}
\hline PROPOSTA & NO DE CAMADAS & TIPO DE LISTA & $\begin{array}{l}\text { CADASTRO DOS } \\
\text { ATUADORES }\end{array}$ & $\begin{array}{c}\text { SISTEMA } \\
\text { OPERACIONAL }\end{array}$ \\
\hline $\begin{array}{c}\text { UBIQUITOUS } \\
\text { HOME CONTROL } \\
\text { AND MONITORING } \\
\text { SYSTEM USING } \\
\text { ANDROID (PIYARE, } \\
\text { 2013) } \\
\end{array}$ & 3 & MANUAL & MANUAL & ANDROID \\
\hline $\begin{array}{c}\text { SAH ( ROY E ROY, } \\
\text { 2014) }\end{array}$ & 2 & MANUAL & MANUAL & ANDROID E IOS \\
\hline $\begin{array}{c}\text { HOUSE } \\
\text { GENIE (ZHONG ET } \\
\text { AL., 2011) }\end{array}$ & 4 & MANUAL & MANUAL & ANDROID E WEB \\
\hline $\begin{array}{l}\text { SMART AUTO HOME } \\
\text { (SATRIA ET AL., } \\
\text { 2015) }\end{array}$ & 2 & MANUAL & MANUAL & $\begin{array}{l}\text { ANDROID, IOS E } \\
\text { WINDOWS PHONE }\end{array}$ \\
\hline $\begin{array}{c}\text { NON-APPLIANCE } \\
\text { CONTROL (DVORAK } \\
\text { ET AL., 2019) }\end{array}$ & 3 & MANUAL & MANUAL & ANDROID \\
\hline $\begin{array}{c}\text { HOME AUTOMATION } \\
\text { SYSTEM (VIKRAM ET } \\
\text { AL., 2017) }\end{array}$ & 3 & MANUAL & MANUAL & ANDROID \\
\hline SCED & 3 & AUTO & AUTO & ANDROID \\
\hline
\end{tabular}

Fonte: Próprio autor

\section{MODELO CONTEXTUAL}

O requisito de simplicidade de uso para um dispositivo como um controle remoto universal só pode ser atendido a partir da captação e tratamento das informações do contexto em que se dá a interação é o usuário e os equipamentos.

A natureza da aplicação é essencialmente contextual: equipamentos podem ser adicionados às residências, ou removidos, o estado dos equipamentos pode ser alterado a partir de comandos dados diretamente nos seus painéis. $O$ crescente da quantidade de equipamentos elétricos na residência exige algum controle do inventário. Por questões de segurança, alguns equipamentos só deverão ser controlados quando o operador estiver próximo, enquanto outros poderão ser controlados remotamente através da Internet. Desta forma, gerenciar adequadamente o contexto é fundamental para o funcionamento adequado do sistema.

A abordagem adotada para o tratamento do contexto é a proposta por Vieira et al. (2011). O sistema tem duas interações para as quais a ciência de contexto é relevante (Focos). São eles:

- Foco 1 (Pessoa, escolher unidade residencial a ser controlada);

- Foco 2 (Pessoa, controlar equipamento)
Esta seção descreve o tratamento de contexto para estes Focos. As Entidades Contextuais são Pessoa, Unidade Residencial e Equipamento. Os Elementos Contextuais destas entidades estão descritos na Tabela 2.

Tabela 2 - Elementos Contextuais das entidades

Table 2 - Contextual elements of the entities

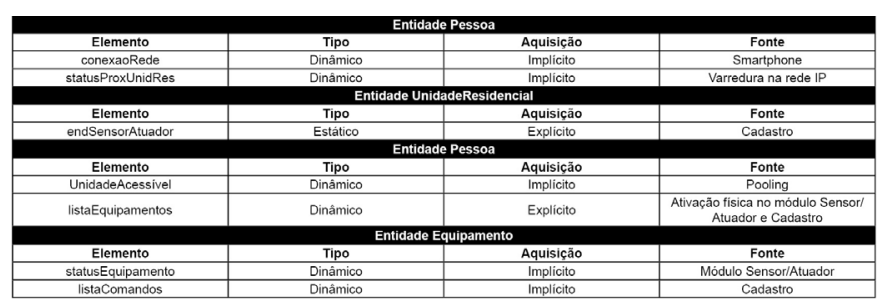

Fonte: Próprio autor

A adaptação ao contexto ocorre nas seguintes situações:

1. A aplicação no dispositivo móvel identifica as Unidades Residenciais às quais o usuário tem acesso para controle e as oferece para seleção. A lista de Unidades Residenciais é previamente cadastrada no sistema, mas a disponibilidade de cada Unidade Residencial depende do seu módulo estar ativo (ligado).

O usuário seleciona a Unidade que deseja controlar.

2. Selecionada uma Unidade Residencial, a aplicação identifica o elemento contextual statusProxUnidRes, que informa se o acesso está sendo feito em proximidade (conexão via rede Wi-fi, com o endereço IP do módulo de Gerência na mesma sub-rede do smartphone) ou à distância (conexão através da Internet).

Se estiver nas proximidades da residência, oferece a relação de todos os equipamentos disponíveis para controle. Se estiver remoto, oferece a lista de equipamentos que podem ser controlados à distância. Esta adaptação ao contexto é descrita no grafo contextual da Figura 1. O usuário então seleciona o Equipamento que deseja controlar.

3. Selecionado o Equipamento, a aplicação apresenta o seu status atual (ligado/desligado) e disponibiliza os comandos que podem ser utilizados para este equipamento. Diferentes equipamentos podem ter conjuntos de controles levemente diferentes. $O$ controle à distância é vedado para alguns tipos de equipamento. Esta adaptação ao contexto está formalizada no grafo contextual da Figura 2. 
Figura 1 - Grafo Contextual do Foco no. 1.

Figure 1 - Contextual Graph of Focus no. 1.

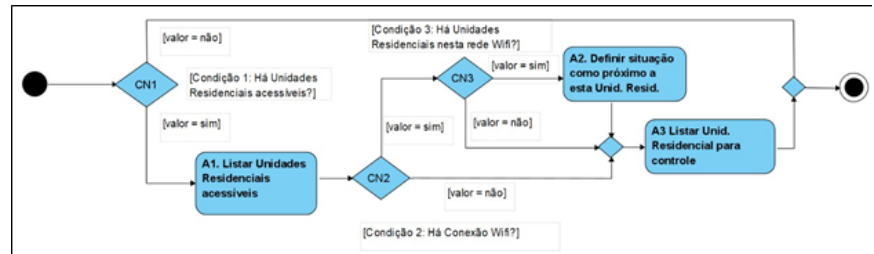

Figura 2 - Grafo Contextual do Foco no. 2

Figure 2 - Contextual Graph of Focus no. 2

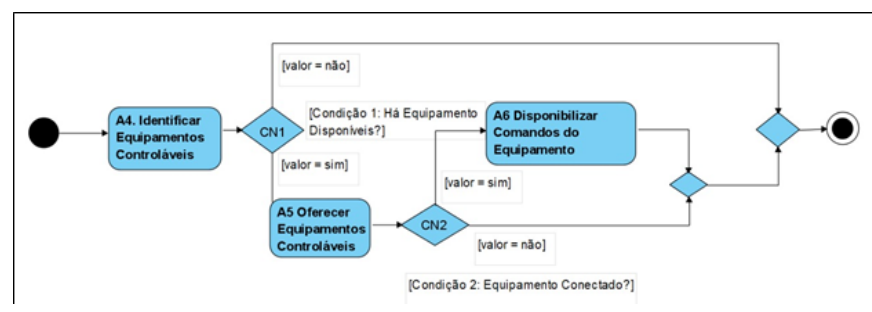

4. Como descrito nos grafos, a aplicação perceberá o posicionamento do usuário em relação à unidade residencial, o conjunto de equipamentos disponíveis para controle e a sua situação, fazendo com que o controle remoto universal seja componente dinâmico que se adaptará às condições mapeadas.

\section{AUTOMAÇÃO RESIDENCIAL COM UM SISTEMA CONTEXTUAL PARA CONTROLE DE EQUIPAMEN- TOS DOMÉSTICOS (SCED)}

O SCED visa possibilitar o monitoramento e controle de equipamentos domésticos através de um smartphone. Os equipamentos podem ser TVs, condicionadores de ar, luzes, cortinas, dispositivos de irrigação, ou quaisquer outros aparelhos elétricos. Haverá uma única tela de controle, independente do equipamento, que disponibilizará quatro comandos básicos (ligar, desligar, aumentar/reduzir a intensidade, mudar opção) e informará a situação do equipamento (ligado/desligado). A tela será oferecida por um aplicativo que será executado no smartphone.

O controle remoto universal do SCED poderá ser usado tanto na proximidade dos equipamentos, quanto remotamente através da Internet, seguindo os padrões definidos pelo modelo desenvolvido por (PIMENTA et al., 2015). Para melhor entendimento, serão descritos os módulos componentes da solução (Interação, Gerência e Atuador) e os recursos utilizados para a construção dessa plataforma.

\section{MÓDULO DE INTERAÇÃO}

Módulo responsável pelo funcionamento do controle remoto universal para no smartphone. É representada por um aplicativo instalado em um dispositivo móvel em que o usuário poderá ter acesso direto ao controle remoto, interagindo através de toques. A partir daí ele poderá controlar todos os dispositivos eletrônicos disponíveis no ambiente.

O funcionamento do controle remoto universal para dispositivos móveis é dividido em dois submodelos (Inicialização e Gerenciamento), que podem ser vistos através da Figura 3. Operam da seguinte forma:

- Submódulo de Inicialização: tem a responsabilidade de realizar a comunicação remota entre o middleware (Gerência) da Unidade Residencial previamente configurada e o controle remoto universal;

- Submódulo de Gerenciamento: responsável pela disponibilidade de uma tela que será utilizada pelo usuário. Este módulo servirá para a interação a ser realizada entre o usuário e os atuadores que estão conectados aos dispositivos eletrônicos disponíveis no ambiente.

Quando o aplicativo do controle remoto universal for iniciado, irá realizar uma busca pela rede local para encontrar o IP do middleware (Gerência). Se o middleware é encontrado na mesma sub-rede, a posição do usuário é considerada como em proximidade. Se o middleware está sendo acessado através da Internet, posição é remota.

Quando ele o encontra o middleware, é feita uma requisição utilizando o protocolo HTTP com o objetivo de achar os dispositivos eletrônicos que estão disponíveis no ambiente. Neste momento, é utilizado JSON (JavaScript Object Notation - Objeto de Notação JavaScript) para organizar as informações dos dispositivos eletrônicos ativos.

Os dados colhidos pelo middleware (Gerência) são repassados para o controle remoto universal sempre que este solicitar, construindo a tela com os dispositivos para visualização do usuário. Quando este escolhe um dos dispositivos disponíveis, será criada uma tela com as características do eletrodoméstico desejado e da posição do usuário em relação à Unidade Residencial. Finalmente, o usuário pode a selecionar botão com a ação desejada para interagir com o dispositivo.

A construção da tela é uma das maiores contribui- 
ções deste trabalho e permite que o indivíduo consiga interagir com os dispositivos que estão espalhados em todo o ambiente. Eles são controlados e representados através de atuadores, que recebem os comandos do middleware (Gerência) e os repassam para o dispositivo eletrônico, que realizará ação desejada.

Quando o eletrodoméstico desejado é selecionado, o controle remoto universal para dispositivos móveis irá realizar uma comunicação através da Wi-Fi com o middleware (Gerência) através do protocolo HTTP, utilizando o seu endereço. O middleware identificará o comando enviado pelo controle universal e o repassará para o Atuador conectado àquele eletrodoméstico. O Atuador irá receber as informações, encaminhando-as para o eletrodoméstico, que realizará ação.

\section{MÓDULO DE GERÊNCIA}

O módulo de gerência faz a intermediação entre os módulos de interação e atuadores. Ele é representado por um aplicativo denominado middleware que irá repassar os comandos do módulo de interação para a os atuadores. Além disso, ele também irá encaminhar as informações advindas dos atuadores para o controle remoto universal dos dispositivos móveis.

As ações realizadas por este módulo estão divididas em três etapas:

- Varredura: é realizada uma busca na rede local para identificar quais são os atuadores existentes no ambiente;

- Processamento: após a finalização da primeira etapa, é feito o processamento das informações referentes aos atuadores;

- Servidor Web: esta etapa caracteriza-se pela inicialização de um servidor Web, que fornece uma lista com as informações dos atuadores no formato JSON.

Quando o middleware, em execução em um Raspberry $\mathrm{Pi}$, é ativado, ele cria um ponto de rede Wi-Fi, que irá funcionar como um ponto de acesso, desta forma os atuadores irão conseguir se comunicar. Depois de terminar a criação da rede, o middleware irá realizar a varredura no ambiente. $O$ segundo passo é iniciado quando os atuadores são encontrados e tem suas informações processadas no middleware, desta forma, é criada uma lista de atuadores no formato JSON que é disponibilizada por meio de um Servidor Web, inicializado pela aplicação.
Os funcionamentos das ações, no módulo de gerência, estão organizados da seguinte maneira: (i) o usuário irá solicitar informações do ambiente através do controle remoto universal para dispositivos móveis, que irá realizar uma requisição ao (ii) middleware, que terá a missão de encaminhar a solicitação para o (iii) atuador realizar a ação comunicando-se com o dispositivo eletrônico.

Desta forma, conclui-se que o módulo de gerência é o centro de toda a plataforma, visto que através dele o controle remoto universal para dispositivos móveis consegue identificar e gerenciar todos os dispositivos espaIhados no ambiente.

\section{MÓDULO ATUADOR}

Este módulo tem como principal função o controle de todos os dispositivos eletrônicos disponíveis no ambiente. Ele é dividido em duas subcamadas, os microcontroladores e os componentes eletrônicos. Estes dispositivos possuem firmwares com programas embarcados para controlar o hardware do atuador.

Os atuadores irão ter dois tipos de funções nesta proposta: função simples que representa comandos mais simples enviados pelo cliente, eles possuem apenas duas variáveis, como ligar e desligar, por exemplo; e funções múltiplas que possui mais de duas variáveis, tal qual as diversas funções existentes para o controle de uma televisão, por exemplo.

Na primeira vez que o atuador é iniciado, ele irá realizar uma conexão através da rede Wi-Fi ao módulo de gerência, que é um middleware. Desta forma o atuador receberá um endereço IP através do DHCP, conforme pode ser visto no passo um. Na segunda etapa, ele irá enviar ao middleware suas principais informações, que são compostas por: endereço IP, identificador, status e nome. Finalmente, o middleware repassa para o servidor Web as novas informações, já atualizadas sobre o dispositivo eletrônico.

Quando o atuador recebe a solicitação do middleware, ele irá identificar qual é a ação e como ela deve ser executada. Após realizar a ação, o atuador irá retornar uma confirmação para o middleware, que irá encaminhar esta informação para o aplicativo universal.

Como um dos objetivos da plataforma SCED é ser de baixo custo, foi necessário encontrar um atuador extremamente barato e eficiente para se comunicar com o 
middleware e os dispositivos eletrônicos no ambiente. Neste caso, o atuador selecionado foi o Arduino, que tem um custo baixo, um módulo Wi-Fi embutido e um microcontrolador ARM acoplado.

\section{APRESENTAÇÃO CONTEXTUAL}

Esta seção tem como principal objetivo a apresentação das funcionalidades do módulo de Interação, bem como a utilização do aplicativo contextual do SCED para dispositivos móveis. Quando o usuário seleciona o botão de ar condicionado, por exemplo, o controle universal irá, contextualmente, construir uma nova tela, em que o usuário terá a possibilidade de controlar algumas das principais funcionalidades do aparelho eletrônico.

No caso do controle para ser utilizado no ar condicionado, existe a seguinte situação: ele possui as opções de ligar, desligar, aumentar e diminuir a temperatura do aparelho. Adicionalmente, informa a situação atual do aparelho, se ligado ou desligado. Além da tela do ar condicionado, o controle universal contextual controla outros equipamentos eletrônicos. Além disso, a tela de lâmpada tem a possibilidade do indivíduo poder ligar o desligar uma lâmpada à distância. Já a tela da televisão permite que o usuário realize uma série de ações e não apenas ligar ou desligar o dispositivo. Através desta tela, o usuário pode aumentar ou diminuir o volume, selecionar a entrada de vídeo com o input, colocar a televisão no mudo ou mudar de canal.

A construção da tela automática feita pelo controle remoto universal é possível como resultado da utilização de técnicas contextuais, que organiza a coleta de informações vitais sobre os dispositivos eletrônicos. O contexto é utilizado para organizar informações sobre qual o atuador, bem como o nome do dispositivo eletrônico que está acoplado a ele e, a partir daí, foi possível carregar o dispositivo na tela do SCED.

\section{AVALIAÇÃo}

O objetivo desta seção é avaliar a plataforma na prática, testar as suas potencialidades e identificar as possíveis limitações. Além disso, pretende-se avaliar a experiência do usuário durante as interações contextuais via celular com o SCED.

Desta maneira, foi elaborado um questionário, construído em conformidade com a unificação de três atributos de usabilidade definido em Schaub et al. (2012). Cada pergunta teve o propósito de avaliar um atributo, conforme relacionado na Tabela 3.

Tabela 3 - Perguntas utilizadas para avaliação do SCED.

Table 3 - Questions used for SCED evaluation.

\begin{tabular}{c|l|l}
\hline Id & \multicolumn{1}{|c|}{ Perguntas } & \multicolumn{1}{|c}{ Atributo } \\
\hline Q1 & $\begin{array}{l}\text { Qual a sua satisfação com o tempo de } \\
\text { resposta do cenário de interação? }\end{array}$ & Eficiência \\
\hline Q2 & $\begin{array}{l}\text { Qual o seu nivel de satisfação com a eficácia } \\
\text { do mecanismo de acionamento e controle de } \\
\text { interação disponibilizado pelo sistema? }\end{array}$ & Eficácia \\
\hline Q3 & $\begin{array}{l}\text { Qual o nivel de satisfação com a facilidade } \\
\text { de uso do sistema? }\end{array}$ & Facilidade de Uso \\
\hline
\end{tabular}

Fonte: Próprio autor

Para coletar as respostas, foi utilizada a escala de Likert (1932), onde as opções de respostas variaram de 1 a 5, sendo 1 - "Concordo Plenamente" e 5 - "Discordo Plenamente". No momento de execução do experimento foram especificados dois documentos: (i) Texto explicativo referente à proposta do trabalho e dicas simples para a utilização do SCED e (ii) um roteiro a ser seguido pelo usuário, contemplando as tarefas a serem executadas no cenário de interação.

A execução e coleta dos dados foi feita com a participação de 4 usuários (todos com mais de 18 anos). O gráfico apresentado na Figura 3 exibe os resultados obtidos após a realização do experimento.

Figura 3 - Resultados do experimento

Figure 3 - Results of the experiment.

- Satisfeito Muto Satisfeto

EFICIÊNCIA

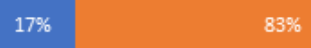

EFICÁCIA

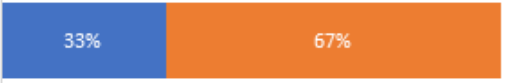

FACILIDADE DE USO

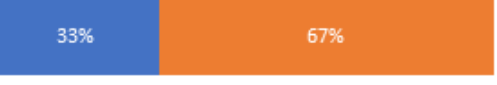

Fonte: Próprio autor

Após análise do gráfico apresentado na Figura 3, percebeu-se que todos os usuários ficaram satisfeitos 
com o SCED, sendo que 33\% ficaram satisfeitos com o sistema e $67 \%$ ficaram muito satisfeitos. Esta foi a mesma numeração para a eficácia do sistema. Finalmente, na métrica de eficiência, a análise apresenta que $17 \%$ dos usuários ficaram satisfeitos e $83 \%$ muito satisfeitos, o que comprova o sucesso da solução apresentada neste artigo.

\section{CONSIDERAÇÕES FINAIS}

Atualmente, o contexto é um elemento importante para o desenvolvimento de uma solução de automação residencial. Desta forma, pode-se ver que os testes de usabilidade demonstraram que, embora a tela criada tenha facilidade de uso, a substituição do controle remoto tradicional por um aplicativo no smartphone enfrenta resistência em função da agilidade.

De fato, a rapidez de manuseio de um controle remoto tradicional é muito maior que o acionamento através do dispositivo móvel, que requer passos como desbloqueio da tela, carga do aplicativo (caso ainda não esteja em execução) ou seleção do aplicativo (se já estiver executando) antes que seja realizada a interação.

Para alguns dispositivos, como a TV, a maior complexidade na interação pode impedir sua adoção. Este fator indica a necessidade de desenvolvimento de uma forma de interação mais ágil, como o caso do uso de um comando por voz, por exemplo. Preservado a abordagem de uma tela genérica, o desenvolvimento de uma interação por voz é uma indicação para pesquisa futura.

Além disso, a necessidade de inventariar, monitorar e controlar à distância equipamentos domésticos, cujo número médio por residência vem crescendo gradualmente e está se tornando evidente. As soluções de mercado verticais (controle remoto genérico fornecido por um fabricante para os seus equipamentos), bem como os caros projetos de automação residencial sob medida deixam um nicho de mercado para um produto voltado especificamente para o controle e que seja de baixo custo. Assim, considera-se que há uma perspectiva concreta de aplicação da solução proposta neste artigo.

\section{REFERÊNCIAS}

BÖHMER, M.; LANDER, C.; KRÜGER, A. What's in the apps for context? extending a sensor for studying app usage to informing context-awareness. In: Proceedings of the 2013 ACM conference on Pervasive and ubiquitous computing adjunct publication. 2013. p. 14231426. DOI: https://doi.org/10.1145/2494091.2496038.

DESAI, N.; KAOWTHUMRONG, K.; LEBSACK, J. et al. Automated selection of remote control user interfaces in pervasive smart spaces. In: Proceedings of the HCIC winter workshop. 2002. p. 1-12.

DVORAK, J.; KREJCAR, O.; CHENG, L. K. Application of universal remote control of non-smart home appliances for smart home concepts. International Journal of Digital Enterprise Technology, v. 1, n. 3, p. 276-291, 2019. DOI: https://doi.org/10.1504/IJDET.2019.097847.

LIKERT, Rensis. A technique for the measurement of attitudes. Archives of psychology, 1932.

PIMENTA, I. G.; SARMENTO, L. N.; KRONBAUER, A. $H$. et al. Integração do Kinect com OpenCV para tratar interações via gestos. In: Proceedings of the 14th Brazilian Symposium on Human Factors in Computing Systems. 2015. p. 123-132.

PIYARE, R. Internet of things: ubiquitous home control and monitoring system using android based smart phone. International journal of Internet of Things, v. 2, n. 1, p. 5-11, 2013. DOI: 10.5923/j.ijit.20130201.02.

ROY, J.; ROY, J. K.; Design of smart universal remote using mobile for home automation. IOSR Journal of Computer Engineering, v. 16, n. 5, p. 73-80, 2014.

SATRIA, A.; PRIADI, M.; LUTHFI, W. L. A. et al. The framework of home remote automation system based on smartphone. International journal of smart home, v. 9, n. 1, p. 53-60, 2015. DOI: 10.14257/ijsh.2015.9.1.06.

SCHAUB, F.; DEYHLE, R.; WEBER, M. I. Password entry usability and shoulder surfing susceptibility on different smartphone platforms. In: Proceedings of the 11th international conference on mobile and ubiquitous multimedia. 2012. p. 1-10.

SU, C. J.; CHIANG, C. Y. Pervasive community care platform: Ambient Intelligence leveraging sensor networks and mobile agents. International Journal of Systems 
VIEIRA, V. A.; TEDESCO, P.; SALGADO, A. C. A. Designing context-sensitive systems: An integrated approach. Expert Systems with Applications, v. 38, n. 2, p. 11191138, 2011.

VIKRAM, N. et al. A low cost home automation system using Wi-Fi based wireless sensor network incorporating Internet of Things (IOT). In: 2017 IEEE 7th International Advance Computing Conference (IACC). IEEE, 2017. p. 174-178.

ZHONG, Y.; SUO, Y.; XUW, Y. U. C. et al. Smart home on smart phone. In: Proceedings of the 13th international conference on Ubiquitous computing. 2011. p. 467-468. DOI: 10.1145/2030112.2030174. 